Original research article

\title{
A study of the hepatoprotective effect of Plantago psyllium L. seed extract against Carbon tetrachloride induced hepatic injury in rats
}

\author{
Mekky M Abouzied 1, 2 *\#, Shaymaa M Mahmoud ${ }^{3}$ *, Ahmed Wahid ${ }^{4}$, Amr E Ahmed ${ }^{3}$, \\ Ahmed M Okasha ${ }^{5}$, Hanan A Soliman ${ }^{6}$, Sultan S Al Thagfan ${ }^{7}$, Eman Z Attia ${ }^{8}$ \\ ${ }^{1}$ Taibah University, College of Pharmacy, Department of Pharmacology and Toxicology, Medina, Kingdom of Saudi Arabia \\ ${ }^{2}$ Minia University, Faculty of Pharmacy, Department of Biochemistry, Minia, Egypt \\ ${ }^{3}$ Beni-Suef University, Faculty of Postgraduate Studies for Advanced Sciences, Biotechnology Department, Beni-Suef, Egypt \\ ${ }^{4}$ Alexandria University, Faculty of Pharmacy, Department of Pharmaceutical Biochemistry, Alexandria, Egypt \\ ${ }^{5}$ Minia University, Faculty of Medicine, Department of Biochemistry, Minia, Egypt \\ ${ }^{6}$ Beni-Suef University, Faculty of Science, Department of Biochemistry, Beni-Suef, Egypt \\ 7 Taibah University, College of Pharmacy, Department of Clinical and Hospital Pharmacy, Medina, Kingdom of Saudi Arabia \\ ${ }^{8}$ Minia University, Faculty of Pharmacy, Department of Pharmacognosy, Minia, Egypt
}

\begin{abstract}
Background: The liver is the main metabolic organ involved in disposal and detoxification of various molecules. Plantago psyllium L. seed has been reported to exert positive effects in some pathological conditions. The current study aims to assess the hepatoprotective effect of Plantago psyllium L. seed extract against carbon tetrachloride-induced hepatotoxicity.

Methods: Male albino Wistar rats were randomly divided into five groups of 10 rats each. Hepatotoxicity was induced by orally administered carbon tetrachloride $\left(\mathrm{CCl}_{4}\right)$ for nine weeks with or without the different treatments which were utilized daily for the whole nine weeks. Serum and tissue samples were then withdrawn and different liver biomarkers were investigated.

Results: Treatment of rats with Psyllium seed ethanolic extract significantly alleviated the toxic effects of $\mathrm{CCl}_{4}$. This was evidenced by its ability to restore liver biomarkers levels. Moreover, treatment with Psyllium seed extract normalized levels of oxidative biomarkers such as lipid peroxidation, hepatic content of reduced glutathione and catalase activity, as well as the expression level of the inflammatory marker TNF-a. Histopathological examination reflected the protective effect of the extract on liver architecture and confirmed the observed biochemical data.

Conclusions: The presented data demonstrates a potential hepatoprotective effect of Psyllium seed extract compared to the standard hepatoprotective drug silymarin. This effect can be attributed to the antioxidant and anti-inflammatory effects of Psyllium extract.
\end{abstract}

Keywords: Anti-oxidant activity; $\mathrm{CCl}_{4}$; Hepatoprotective activities; Plantago psyllium L.; TNF- $\alpha$

\section{Highlights:}

- $\mathrm{CCl}_{4}$ induced a hepatotoxic effect on the biochemical and the histological level.

- Psyllium seed extract (PSE) exerted a hepatoprotective effect.

- It restored catalase activity and reduced glutathione content, and ameliorated lipid peroxidation.

- It alleviated the histopathological signs of $\mathrm{CCl}_{4}$-induced hepatotoxicity.

- PSE effects can be regarded to its antioxidant properties.

- PSE is a good candidate as a phytotherapeutic agent to protect the liver.

\section{Abbreviations:}

$\mathrm{CCl}_{4}$, carbon tetrachloride; RE, Rutin equivalent; GAE, gallic acid equivalent; DPPḦ, 2, 2‘-diphenyl-1-picrylhydrazyl; TNF- $\alpha$, Tumor necrosis factor alpha; AST, Aspartate transaminase; ALT, Alanine transaminase; GSH, glutathione; LDH, Lactate dehydrogenase; MDA, malondialdehyd; ALP, Alkaline phosphatase; TG, triglyceride; CAT, catalase; PSE, Psyllium seed extract; ROS, reactive oxygen species; TBARS, thiobarbituric acid reactive substance

\footnotetext{
* Author for correspondence: Mekky M Abouzied, Taibah University, College of Pharmacy, Department of Pharmacology and Toxicology, Medina, Kingdom of Saudi Arabia; e-mail: m_meky2001@yahoo.com

\# Both authors contributed equally to the manuscript.

http://doi.org/10.32725/jab.2020.006

Submitted: 2019-04-23 • Accepted: 2020-04-28 • Prepublished online: 2020-05-21

J Appl Biomed 18/2-3: 80-86 • EISSN 1214-0287 • ISSN 1214-021X

c 2020 The Authors. Published by University of South Bohemia in České Budějovice, Faculty of Health and Social Sciences.

This is an open access article under the CC BY-NC-ND license.
} 


\section{Introduction}

Despite the fact that various hepatic issues are in charge of a remarkable number of liver transplantations and deaths recorded around the world, accessible therapeutic alternatives are extremely limited. This provoked an extraordinary interest in the improvement of new powerful drugs (Eidi et al., 2012).

Plantago psyllium L. (Plantaginaceae), popularly known as 'Psyllium', is a plant native to tropical regions (Devesh et al., 2014). Its seeds were used as a thickening agent for manufacturing tablets as they contain pentoses, hexoses, and uronic acids (Gelissen et al., 1994). Some earlier studies reported antioxidant activity and a positive effect in the treatment of inflammatory bowel disease for Plantago (Kardosova and Machova, 2006; Rodriguez-Cabezas et al., 2003). Recently, phytochemical studies have attributed these biological activities to the content of fixed oils, flavonoids, tannin, aucubin glycoside (iridoid), sugars, sterols and proteins as well as the presence of hydrocolloidal polysaccharide (mucilage) in the outer seed coat (Devesh et al., 2014).

Oxidative stress that results from free radicals overburdening the endogenous antioxidant mechanisms can lead to cellular damage and tissue injury representing a main cause for various disorders including liver dysfunction ( $\mathrm{Li}$ et al., 2015). There is an increasing amount of evidence that natural antioxidants such as polyphenols and flavonoids have a crucial role in protecting cells against oxidative damage. Their free radical scavenging abilities support the concept of using these products in the treatment of a wide range of disorders (Pandey and Rizvi, 2009).

Carbon tetrachloride $\left(\mathrm{CCl}_{4}\right)$ is a known hepatotoxin that is biotransformed by hepatic microsomal CYP450 producing highly reactive free radicals. These metabolites induce a state of lipid peroxidation and oxidative stress (Brautbar and Williams, 2002). It has been shown before that antioxidants protect against $\mathrm{CCl}_{4}$ induced-hepatotoxicity via enhancing endogenous antioxidant capacity and inhibiting lipid peroxidation (Singal et al., 2011). Although the antioxidant activity of P. psyllium seeds had been reported earlier, its hepatoprotective effects have not yet been investigated (Patel et al., 2016). In this current work we aimed at investigating the hepatoprotective effects of $P$. psyllium seeds extract against $\mathrm{CCl}_{4}$ induced-hepatotoxicity.

\section{Materials and methods}

\section{Source of Plantago psyllium L. seed}

P. psyllium seeds (batch No.: 11315302000471, gluten-free, pesticide-free, GMO-free and raw) used in this study were purchased from a local supplier in Cairo, Egypt.

\section{Source of animals}

Male albino Wistar rats (200 $\pm 5 \mathrm{~g}$ ) were purchased from Faculty of Veterinary Medicine, Minia University.

\section{Institutional animal care and use committee}

Animal experiments were conducted following the guidelines for the care and use of laboratory animals of the National Institutes of Health (NIH publication No. 85-23, revised 1985). All experimental procedures of the study were approved by the Animal Care and Use Committee of Biotechnology department, Faculty of postgraduates for advanced sciences, Beni-Suef University.

\section{Chemicals}

All chemicals were of analytical grade. Chemicals and solvents were purchased from Sigma (USA) and Merck (Germany) respectively. Different parameters evaluated in the present study were assessed using commercial kits following the manufacturer's instructions.

\section{Extract preparation}

Dried seeds (500 g) were suspended in two liters of $70 \%$ ethanol at $25{ }^{\circ} \mathrm{C}$ with continuous shaking for $48 \mathrm{~h}$. The process was repeated two times and the pooled extract was then concentrated under reduced pressure in order to yield P. psyllium seeds ethanolic extract (PSE); a semi-solid residue that was used for the experiments (Kil et al., 2009).

\section{DPPH radical scavenging activity assay}

Free radical scavenging activity of PSE was measured using DPPH (Brand-Williams, 1995). The concentration of the extract that provided $50 \%$ inhibition $\left(\mathrm{EC}_{50}\right)$ was then calculated. Percentage of DPPH inhibition was calculated using the formula:

$\%$ of inhibition $=100 \times[1-($ Absorbance with compound $/ \mathrm{Ab}$ sorbance of the blank)].

\section{Estimation of total phenolic and flavonoids content}

The content of total phenolic compounds in PSE was evaluated by Folin-Ciocalteu method (Singleton et al., 1999) using gallic acid as a standard. Briefly, $500 \mu \mathrm{l}(25 \mathrm{mg} / \mathrm{ml})$ of PSE extract were added to $2.5 \mathrm{ml}$ of Folin-Ciocalteu reagent $(0.2 \mathrm{M})$ and allowed to stand for $4 \mathrm{~min}$. After that, the volume was filled up to $5 \mathrm{ml}$ with saturated sodium carbonate and the absorbance was measured after $2 \mathrm{~h}$ incubation at RT at $760 \mathrm{~nm}$. As a standard, gallic acid was used and the results were expressed as mg gallic acid equivalents/g dry weight. Evaluation of total flavonoids was conducted following the method of Park et al. (2008), using rutin as a standard.

\section{Animals and treatment}

\section{Toxicity study for PSE}

A group of 60 Wistar male and female rats were assigned into 6 groups of 10 rats each ( 5 males and 5 females). The first group received olive oil per oral gavage for 28 days. The remaining groups received 50, 100, 200, 500 and $1000 \mathrm{mg} / \mathrm{kg}$ of PSE in olive oil daily for the same period to test the safety of the extract.

\section{Experimental design}

Male albino Wistar rats were housed in the animal house of the faculty of Pharmacy, Beni-Suef University. The rats were kept in plastic cages and supplied with standard animal chow and water ad libitum at $25 \pm 2{ }^{\circ} \mathrm{C}$ and $12 \mathrm{~h}$ dark/light cycle.

Fifty rats were categorized into five groups of 10 rats per group. Carbon tetrachloride $\left(\mathrm{CCl}_{4}\right)$ as well as the drugs used for treating the rats were administered for a period of 9 weeks as follows:

Group I (Normal healthy control): rats received olive oil per oral gavage twice a week for the whole period of the experiment.

Group II ( $\mathrm{CCl}_{4}$-intoxicated group): rats received $0.8 \mathrm{ml} / \mathrm{kg}$ body weight $\mathrm{CCl}_{4}$ /olive oil per oral gavage twice a week to induce hepatic injury.

Group III (silymarin-treated group): rats received $0.8 \mathrm{ml} / \mathrm{kg}$ body weight $\mathrm{CCl}_{4}$ /olive oil per oral gavage twice a week along with a daily oral dose of silymarin (100 mg/kg of bodyweight). 
Group IV (PSE-treated group): Rats were given $\mathrm{CCl}_{4}$ as in group II and a daily dose of PSE (100 mg/kg bodyweight, in olive oil).

Group V (Normal Feed): rats received PSE only $(100 \mathrm{mg} / \mathrm{kg}$ of bodyweight) beside their normal diet without $\mathrm{CCl}_{4}$ (El-Sayed et al., 2015).

\section{Sample collection}

By the end of the experiment, animals were sacrificed by cervical decapitation after light ether anesthesia, and blood was collected by cardiac puncture method. Blood samples were permitted to clot for 20-30 minutes at room temperature, then serum was separated by centrifugation at $2000 \times \mathrm{g}$ for $20 \mathrm{~min}$, and stored at $-20{ }^{\circ} \mathrm{C}$ until needed. Liver from animals of the different groups were promptly collected. From each liver, a part was snap-frozen for tissue homogenization and another part (from the right distal lobe) was rinsed in saline and fixed in formalin solution and processed for further histological examination.

\section{Biochemical analysis}

The collected serum samples were used for the estimation of the biochemical parameters (aminotransferases (ALT and AST), alkaline phosphatase (ALP), serum albumin, triglycerides (TG), cholesterol, total bilirubin, urea and creatinine) using commercial assay kits (Biodiagnostics, Egypt).

\section{Estimation of lipid peroxidation, reduced glutathione and catalase activity}

Lipid peroxidation measured as malondialdehyde equivalent (MDA), reduced glutathione (GSH) content and catalase (CAT) activity were estimated using commercially available kits following the manufacturer's instructions.

\section{Assessment of TNF- $\alpha$ serum level}

Analysis of serum samples for their content of TNF- $\alpha$ was performed using a commercially available ELISA kit (ORiGENE, cat: EA100366) as per manufacturer's instructions.

\section{Histopathological studies}

Formalin fixed liver tissues were dehydrated in a series of increasing alcohol concentrations and cleared in xylol before being embedded in paraffin wax. A rotary microtome was used to cut $5 \mu \mathrm{m}$-thick sections which were then stained with hematoxylin and eosin (H\&E). Liver specimens were anonymously examined under a light microscope-joined Nikon camera (E400). A scoring system was used for assessing the effect of the different treatments (Desmet, 2003; French et al., 1988). The scoring followed the following scale:

Score $0=$ no visible cell damage

Score 1 = focal hepatocyte damage on less than $25 \%$ of the tissue

Score 2 = focal hepatocyte damage on $25-50 \%$ of the tissue

Score 3 = extensive, but focal, hepatocyte lesions

Score 4 = global hepatocyte necrosis

\section{Statistical analysis of the data}

Data are expressed as mean \pm SEM, and analyzed by one-way analysis of variance (ANOVA) test followed by Dunnett and Tukey post-hoc test. $P$-value $<0.05$ was considered to show significant differences for all parameters using the Graph Pad Prism 6 software followed by Tukey-Kramer test (Version 6.00 for Windows, GraphPad Software, San Diego, California, USA).

\section{Results}

\section{Toxicity study for PSE}

Animals receiving variable doses of PSE did not show morbidity, signs of toxicity or changes in animal behavior compared to the healthy control animals. Analysis of serum hepatic and renal biomarkers (ALT, AST, bilirubin and albumin, urea and creatinine) showed that a dose up to $1000 \mathrm{mg} / \mathrm{kg}$ of PSE is safe and can be administered without any side effects (data not shown).

\section{In vitro $D P P H$ radical scavenging activity of $P S E$}

DPPH radical scavenging activity is a widely used assay to evaluate the antioxidant activity of natural drugs, where a direct correlation between the antioxidant capacity and the reduction in DPPH absorbance is evaluated (Wahid et al., 2016). In this study, the free radical scavenging activity of different concentrations of PSE was estimated based on diminished DPPH absorbance, using ascorbic acid as a standard antioxidant. Antioxidant activity of $1 \mathrm{mg} / \mathrm{ml}$ PSE was $88.33 \%$, whereas the antioxidant activity of the same concentration of ascorbic acid was $92.78 \%$ (Fig. 1).

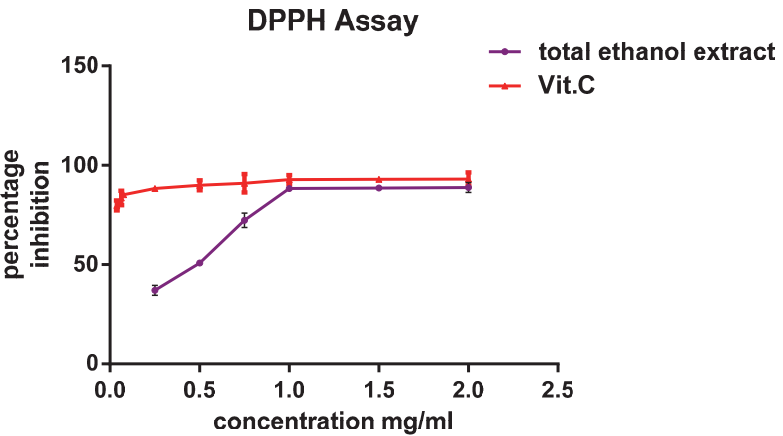

Fig. 1. DPPH scavenging activity of different concentrations of P. psyllium seed ethanolic extract compared to ascorbic acid as a standard DPPH radical scavenger $(n=3)$

\section{Total phenolic and flavonoid contents}

Using the constructed calibration curves $\left(R^{2}=0.998\right)$, total phenolic content of PSE was calculated as $16.17 \pm 0.03 \mathrm{mg}$ gallic acid equivalent/g dried weight, and the total flavonoid content $\left(R^{2}=0.999\right)$ was calculated as $1.9 \pm 0.1 \mathrm{mg}$ rutin equivalent/g dried weight.

\section{Effect of PSE treatment on serum ALT, AST and ALP}

As observed in Table 1, treating animals with $\mathrm{CCl}_{4}$ resulted in a significant elevation of liver aminotransferases and ALP activity compared to healthy control. Interestingly, co-administration of PSE with $\mathrm{CCl}_{4}$ protected against $\mathrm{CCl}_{4}$-induced hepatocellular damage as observed in the form of a significant reduction of enzyme activities compared to $\mathrm{CCl}_{4}$ group. The effect of PSE was comparable to that achieved by silymarin which also resulted in a significantly lower levels of ALT, AST and ALP enzymes compared to $\mathrm{CCl}_{4}$-treated group. Animals receiving PSE showed a non-significant difference in the levels of ALT, AST and ALP compared to the normal healthy control group. 
Table 1. Effects of the different treatments $(100 \mathrm{mg} / \mathrm{kg})$ on serum and liver biochemical indices in $\mathrm{CCl}_{4}$-induced hepatotoxicity in rats

\begin{tabular}{lccccc}
\hline & Control & $\mathrm{CCl}_{4}$ & $\mathrm{CCl}_{4}+$ silymarin & $\mathrm{CCl}_{4}+\mathrm{PSE}$ & PSE \\
\hline AST (U/l) & $22.25 \pm 1.74$ & $299.3 \pm 30.68^{\mathrm{a}}$ & $236.6 \pm 14.95^{\mathrm{c}}$ & $110.5 \pm 7.97^{\mathrm{c}}$ & $25.86 \pm 1.94$ \\
ALT (U/l) & $25.55 \pm 2.20$ & $298.5 \pm 20.14^{\mathrm{a}}$ & $155.8 \pm 13.70^{\mathrm{c}}$ & $63.37 \pm 6.99^{\mathrm{c}}$ & $25.08 \pm 1.18$ \\
ALP (U/l) & $7.04 \pm 0.52$ & $9.52 \pm 0.25^{\mathrm{a}}$ & $8.69 \pm 0.32^{\mathrm{c}}$ & $7.46 \pm 0.39^{\mathrm{c}}$ & $7.09 \pm 0.47$ \\
Bilirubin (mg/dl) & $0.44 \pm 0.04$ & $0.77 \pm 0.05^{\mathrm{a}}$ & $0.75 \pm 0.04^{\mathrm{c}}$ & $0.48 \pm 0.02^{\mathrm{c}}$ & $0.40 \pm 0.02$ \\
Albumin (mg/dl) & $4.92 \pm 0.17$ & $3.73 \pm 0.20^{\mathrm{b}}$ & $4.32 \pm 0.30^{\mathrm{d}}$ & $4.65 \pm 0.13^{\mathrm{c}}$ & $4.79 \pm 0.11$ \\
Cholesterol (mg/dl) & $115.0 \pm 18.59$ & $236.8 \pm 6.50^{\mathrm{a}}$ & $228.9 \pm 8.76^{\mathrm{c}}$ & $204.3 \pm 20.58^{\mathrm{c}}$ & $117.9 \pm 13.52$ \\
Triglycerides (mg/dl) & $97.84 \pm 8.78$ & $143.9 \pm 9.91^{\mathrm{b}}$ & $134.7 \pm 8.98^{\mathrm{d}}$ & $121.5 \pm 11.57^{\mathrm{d}}$ & $99.82 \pm 7.17$ \\
Urea (U/l) & $31.77 \pm 2.14$ & $54.06 \pm 3.97^{\mathrm{a}}$ & $46.38 \pm 1.85^{\mathrm{c}}$ & $32.00 \pm 2.46^{\mathrm{c}}$ & $31.78 \pm 1.75$ \\
Creatinine (mg/dl) & $0.84 \pm 0.07$ & $1.85 \pm 0.05^{\mathrm{a}}$ & $1.43 \pm 0.16^{\mathrm{c}}$ & $0.84 \pm 0.07^{\mathrm{c}}$ & $0.81 \pm 0.06$ \\
\hline
\end{tabular}

Experiments were done in triplicates, and the standard deviation was then calculated $(n=10) ;{ }^{\mathrm{a}} p<0.01 ;{ }^{\mathrm{b}} p<0.001$ compared to healthy control; ${ }^{c} p<0.01 ;{ }^{d} p<0.001$ compared to $\mathrm{CCl}_{4}$-treated. Statistically significant in the Duncan test.

\section{Effect of PSE on serum lipid profile, albumin and total bilirubin levels}

Table 1 shows the effect of PSE on serum levels of cholesterol, $\mathrm{TG}$, total bilirubin and albumin. $\mathrm{CCl}_{4}$ intoxication significantly increased the serum lipids and total bilirubin $(P<0.0001)$. In contrast, albumin level was found to decrease significantly in this group compared to the healthy control group of rats $(P<$ 0.001). Interestingly, similar to silymarin, PSE treatment significantly prevented $\mathrm{CCl}_{4}$-induced alterations of these serum parameters compared to $\mathrm{CCl}_{4}$-treated group.

\section{Effect of PSE on GSH, catalase activity and lipid peroxidation (MDA)}

Liver homogenates of $\mathrm{CCl}_{4}$ intoxicated group showed a significantly decreased GSH level and catalase activity (Fig. 2A, C, $p<0.0001$ ), whereas a significant increase in lipid peroxidation measured as MDA was observed (Fig. 2B, $p<0.05$ ). Interestingly, $\mathrm{CCl}_{4}$-induced GSH and catalase depletion and increased lipid peroxidation were significantly alleviated upon treatment with PSE ( $p<0.0001, p<0.0001$ and $p<0.05$, respectively) or silymarin.

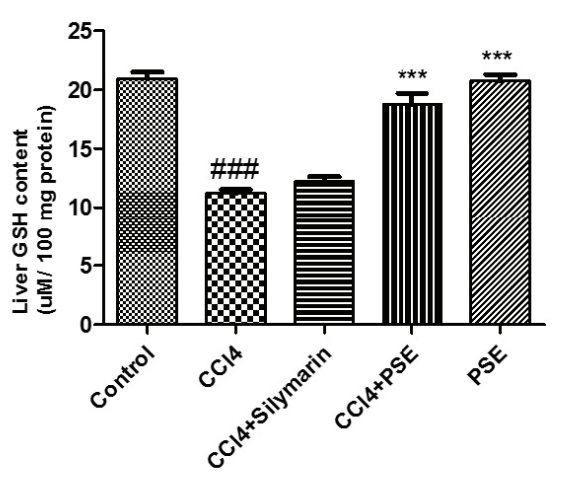
Control
EII CCl4
E CCl4+Silymarin
III CCl4+PSE
שIIJ PSE

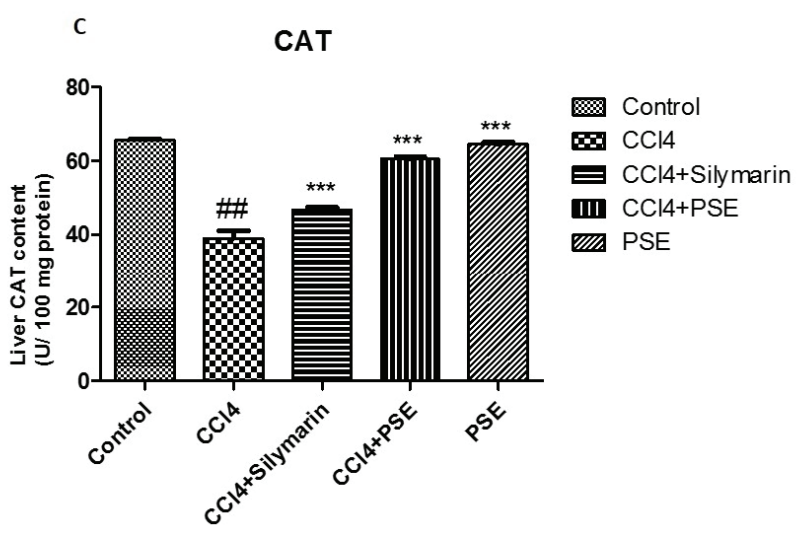

Fig. 2. Effect of the different treatments on the hepatic antioxidant capacity measured as (A) total hepatic glutathione content, (B) lipid peroxidation and $(\mathbf{C})$ catalase activity in liver tissue $(n=10) . \mathrm{CCl}_{4}$ induced lipid peroxidation measured as MDA and depleted GSH and catalase activity compared to healthy group. PSE treatment significantly restored GSH stores and catalase activity and normalized MDA levels compared to $\mathrm{CCl}_{4}$ group. \#\# $p<0.001$; \#\#\# $p<0.0001$ compared to healthy control; ${ }^{*} p<0.05 ;{ }^{* * *} p<0.0001$ compared to CCl . 


\section{Effect of PSE on the expression level of serum TNF- $\alpha$}

We have investigated the variation in the expression level of TNF- $\alpha$ among the different test groups. As shown in Fig. 3, intoxication with $\mathrm{CCl}_{4}$ resulted in a significant up-regulation of TNF- $\alpha$ expression compared to healthy control groups (***, $p<0.0001$ ). Administration of PSE or silymarin in $\mathrm{CCl}_{4}$-intoxicated rats resulted in a significant reduction of TNF- $\alpha$ expression compared to its expression after $\mathrm{CCl}_{4}$ treatment (\#\#\#, $p<0.0001$ ). No significant difference could be detected between silymarin and PSE treatment after $\mathrm{CCl}_{4}$ intoxication. Also no significant difference in TNF-a expression was observed between control animals and animals that received PSE alone $(p>0.05)$.

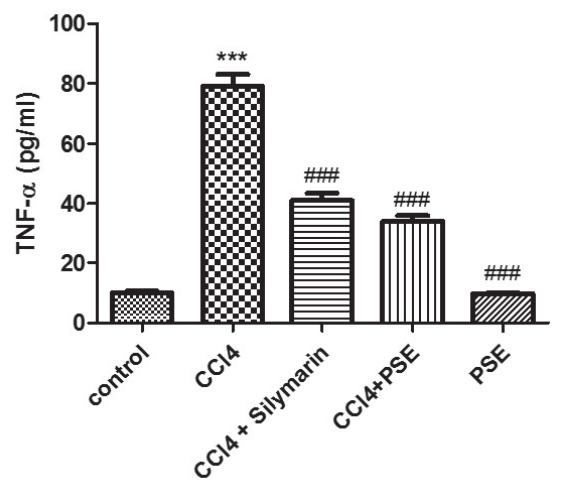

Fig. 3. Serum TNF- $\alpha$ levels after the different treatments. Values are presented as mean \pm SEM, $n=10$ per group. *** Significantly different $(p<0.0001)$ from the control group. \#\#\# Significantly different $(p<0.0001)$ from $\mathrm{CCl}_{4}$-treated group.

\section{Histopathological examination}

Histopathological investigations were performed for H\&Estained liver sections of animals from the different test groups to assess the effect of the different treatments. As can be observed in Fig. 4A, healthy control animals as well as those treated with PSE alone showed normal hepatic architecture with no marked histopathological manifestations (Fig. 4A, E), whereas, liver specimens from $\mathrm{CCl}_{4}$-treated animals showed marked hepatocellular damage in the form of centrilobular and vacular degeneration along with lymphocyte invasion, disruption of hepatic cords and necrosis (Fig. 4B). Treating $\mathrm{CCl}_{4}$-intoxicated animals with PSE effectively protected against $\mathrm{CCl}_{4}$-induced damage, where the hepatic tissue showed absence of lymphocytes and minimal signs of degeneration and necrosis (Fig. 4D). The sections from PSE group were comparable to those of silymarin treated animals (Fig. 4C). Fatty degeneration, necrosis, inflammation and cell swelling were evaluated in sections from the different groups and scored as shown in Fig. $4 \mathrm{~F}$. $\mathrm{CCl}_{4}$ treatment induced a significant increase in the score of all four parameters compared to the healthy control administering PSE $(p<0.001)$, whereas treating $\mathrm{CCl}_{4}$ intoxicated animals with PSE or silymarin significantly improved the signs of tissue damage as observed by reduced scoring $(p<0.001)$.

\section{Discussion}

Carbon tetrachloride is a commonly used substance to induce experimental liver toxicity that is comparable to human hepatotoxicity. It becomes basically metabolized in the endoplasmic reticulum of hepatocytes via CYP450 enzymes producing free radicals. These radicals covalently bind to polyunsaturat-
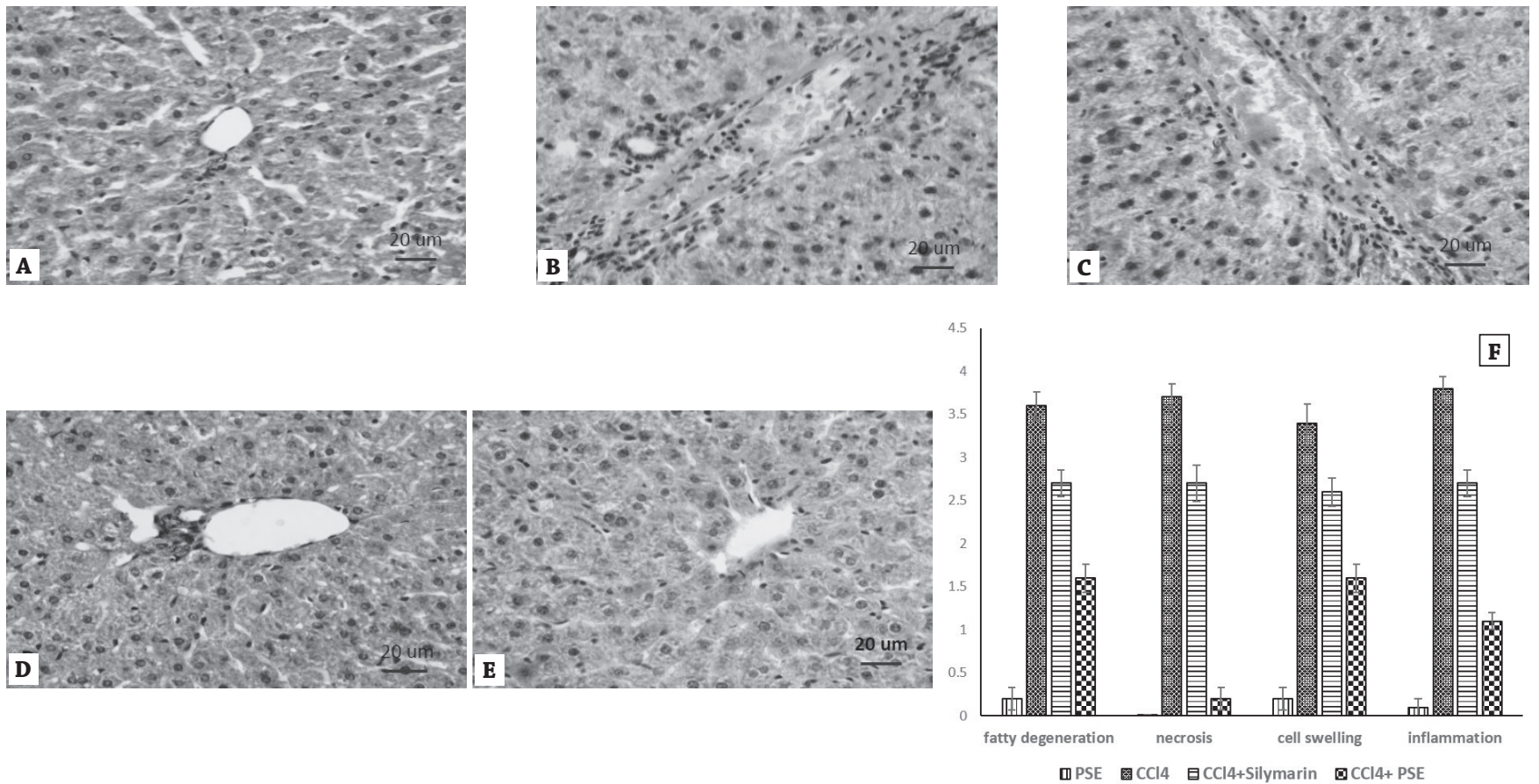

Fig. 4. Histopathological investigation of H\&E-stained liver sections from the different test groups. (A) Liver section of normal control showing normal central vein and hepatocytes. (B) Liver section of $\mathrm{CCl} 4$ animals showing centrilobular necrosis and extensive fatty changes. (C) Liver section from $\mathrm{CCl}_{4}$-silymarin-treated animals showing mild congestion and swelling of liver cells with minimal necrosis. (D) Liver section from $\mathrm{CCl}_{4}$-psyllium extract-treated animals reflecting minimal lobular inflammation and mild congestion. (E) Liver section from $P$. psyllium seedtreated animals showing normal unaffected architecture. Scale bar is $20 \mu \mathrm{m}$. (F) Scoring of liver damage parameters, $n=10$. 
ed fatty acids and induce oxidative damage to hepatic cellular membranes. They also attack a wide range of biomolecules leading to DNA strand breaks and lipid peroxidation, which is one of the standard causes of biomembrane degradation (Diao et al., 2011). It has been reported that free radical scavenging is one of the main mechanisms that protect against oxidative degradation of lipids (Singal et al., 2011; Umamaheswari and Chatterjee, 2008).

In the current study, we were able to show that oral administration of PSE possesses a hepatoprotective potential that was confirmed by biochemical and histopathological investigations as well. The extract of $P$. psyllium seed showed a strong antioxidant capacity which could be regarded to the high phenolic and flavonoid content. These active constituents are known to contribute to the antioxidant potential of natural drugs (Fernandez-Panchon et al., 2008).

In this study, serum level was assessed in the different groups for AST, ALT and ALP, which are widely used biomarkers to assess liver functions. They are cytosolic enzymes that become released into the systemic circulation upon injury to cell membranes, indicating hepatocellular damage (Gowda et al., 2009). Intoxication with $\mathrm{CCl}_{4}$ caused a significant increase of these enzymes' levels compared to control animals, which coincides with previous studies reporting similar findings (Huang et al., 2012). In addition, $\mathrm{CCl}_{4}$ administration negatively altered the lipid profile of the corresponding animals in the form of increased serum cholesterol and triglycerides. Administration of PSE effectively protected against $\mathrm{CCl}_{4}$-induced damage and kept the levels of these enzymatic markers comparable to control animals and normalized the level of serum lipids. This protective effect of PSE can be attributed to the antioxidant effect of the extract which provides enough membrane stabilization for the hepatocytes, hence antagonizing leakage of liver enzymes into the cytosol (Anosike et al., 2018). These results are in line with those reported by other groups indicating the effectiveness of antioxidants in such cases (Feng et al., 2011; Hung et al., 2006).

Tissue damage is initiated soon after depletion of GSH, which is supposed to provide protection against free radicals and oxidative stress. Therefore, the decreased levels of GSH in liver tissues of $\mathrm{CCl}_{4}$-intoxicated rats in the current study reflect oxidative damage to liver tissues. On the other hand, the antioxidant capacity of $P$. psyllium seed extract scavenged $\mathrm{CCl}_{4}$-induced free radicals and protected against depletion of GSH level. These results are in line with previous reports that showed significant depletion of hepatic GSH content upon $\mathrm{CCl}_{4}$ intoxication and restoration of its content after administration of different extracts with antioxidant properties (Huang et al., 2012; Hung et al., 2006).

Thiobarbituric acid reactive substance (TBARS) is used as a biomarker for estimation of lipid peroxidation. Lipid peroxidation indicates damage to cellular membranes and the disability of the antioxidant defense system. $\mathrm{CCl}_{4}$-induced oxidative stress results in the production of massive amounts of free radicals that interact with polyunsaturated fatty acids (PUFAs) in hepatic tissues, leading to their peroxidation that is detected by the up-regulated TBARS levels (Chang et al., 2009). In agreement with this, $\mathrm{CCl}_{4}$ administration induced lipid peroxidation in animals' hepatic tissues, whereas concurrent administration PSE significantly ameliorated lipid peroxidation as observed in the form of normalized MDA levels. This positive effect of the extract on lipid peroxidation can be ascribed to the powerful antioxidant and free radical scavenging activities of the extract. These results are in concurrence with previous reports that proved the ability of antioxidants to alleviate
$\mathrm{CCl}_{4}$-induced hepatotoxicity by inhibiting lipid peroxidation (Elgawish et al., 2015; Teselkin et al., 2000).

Catalase (CAT) is a widely distributed enzyme that decomposes hydrogen peroxide and hence protects the tissues from profound damaging hydroxyl free radicals (Chance et al., 1952). Excessive exposure to free radicals, as in case of $\mathrm{CCl}_{4}$ intoxication, diminishes the activity of CAT and consequently brings about various harmful impacts in the hepatic tissue. These results are in agreement with Abouzied et al., who reported a decreased catalase activity upon $\mathrm{CCl}_{4}$ administration (2016). Interestingly, PSE restored catalase enzyme activity as observed in the current study. This effect of the extract can be regarded to its antioxidant capacity, which scavenges $\mathrm{CCl}_{4}$-induced free radicals and hence saves catalase activity from being depleted.

TNF- $\alpha$ is a pro-inflammatory cytokine that plays a crucial role in regulating many physiological events, and is considered as a marker for the inflammatory condition. It is produced by Kupffer cells upon $\mathrm{CCl}_{4}$-mediated tissue damage inducing the expression of further cytokines as reported earlier (Diao et al., 2011). In agreement with this and other reports, $\mathrm{CCl}_{4}$ intoxication in the present study leads to severe inflammation and hence an over expression of TNF- $\alpha$ gene (Abouzied et al., 2016). Fortunately, P. psyllium extract effectively ameliorated $\mathrm{CCl}_{4}$-induced inflammation and hence TNF- $\alpha$ expression, indicating a healing effect for the PSE on liver cells via modulating the cytokine expression and the underlying inflammatory process.

\section{Conclusions}

The data presented in the current study present a proof for the hepatoprotective activity of $P$. psyllium seed extract. This effect can be regarded to the high content of flavonoids and phenolic compounds which possess a strong antioxidant, radical scavenging and anti-inflammatory effects, protecting the hepatic tissue against $\mathrm{CCl}_{4}$-induced damage. This introduces $P$. psyllium seed extract as a promising therapeutic candidate against oxidative stress-mediated liver disorders.

\section{Conflict of interests}

The authors declare that they have no conflict of interests.

\section{Funding}

This research did not receive any specific grant from funding agencies in the public, commercial, or not-for-profit sectors.

\section{Acknowledgement}

We thank Dr. Heba Eltahir for revising and editing the manuscript.

\section{References}

Abouzied MM, Eltahir HM, Taye A, Abdelrahman MS (2016). Experimental evidence for the therapeutic potential of tempol in the treatment of acute liver injury. Mol Cell Biochem 411: 107-115. DOI: 10.1007/s11010-015-2572-2.

Anosike CA, Igboegwu ON, Nwodo OFC (2018). Antioxidant properties and membrane stabilization effects of methanol extract of Mucuna pruriens leaves on normal and sickle erythrocytes. J Tradit Complement Med 9(4): 278-284. DOI: 10.1016/j. jtcme.2017.08.002. 
Brand-Williams W, Cuvelier ME, Berset C (1995). Use of a free radical method to evaluate antioxidant activity. LWT - Food Sci Technol 28: 25-30. DOI: 10.1016/S0023-6438(95)80008-5.

Brautbar N, Williams J 2nd (2002). Industrial solvents and liver toxicity: risk assessment, risk factors and mechanisms. Int J Hyg Environ Health 205(6): 479-491. DOI: 10.1078/1438-463900175.

Chance B, Greenstein DS, Roughton FJW (1952). The mechanism of catalase action. I. Steady-state analysis. Arch Biochem Biophys 37(2): 301-321. DOI: 10.1016/0003-9861(52)90194-X

Chang CY, Chen YL, Yang SC, Huang GC, Tsi D, Huang CC, et al. (2009). Effect of schisandrin B and sesamin mixture on $C C l(4)$ induced hepatic oxidative stress in rats. Phytother Res 23(2): 251-256. DOI: 10.1002/ptr.2602.

Desmet VJ (2003). Liver tissue examination. J Hepatol 39: S43-49. DOI: 10.1016/S0168-8278(03)00138-7.

Devesh T, Nishat A, Tripathi Y (2014). Phytochemistry and pharmacology of Plantago ovata: A natural sourse of laxative medicine. World J Pharm Res 3: 361-372. DOI: 0.13140/2.1.5043.4885.

Diao Y, Zhao XF, Lin JS, Wang QZ, Xu RA (2011). Protection of the liver against $\mathrm{CCl}_{4}$-induced injury by intramuscular electrotransfer of a kallistatin-encoding plasmid. World J Gastroenterol 17: 111-117. DOI: 10.3748/wjg.v17.i1.111.

Eidi A, Mortazavi P, Bazargan M, Zaringhalam J (2012). Hepatoprotective activity of cinnamon ethanolic extract against $\mathrm{CCl}_{4}$-induced liver injury in rats. EXCLI J 11: 495-507. DOI: $10.17877 / \mathrm{DE} 290 \mathrm{R}-4957$

El-Sayed YS, Lebda MA, Hassinin M, Neoman SA (2015). Chicory (Cichorium intybus L.) root extract regulates the oxidative status and antioxidant gene transcripts in $\mathrm{CCl}_{4}$-induced hepatotoxicity. PLoS One e0121549. DOI: 10.1371/journal.pone.0121549.

Elgawish RAR, Rahman HGA, Abdelrazek HMA (2015). Green tea extract attenuates $\mathrm{CCl}_{4}$-induced hepatic injury in male hamsters via inhibition of lipid peroxidation and p53-mediated apoptosis. Toxicol Rep 2: 1149-1156. DOI: 10.1016/j.toxrep.2015.08.001.

Feng Y, Wang N, Ye X, Li H, Feng Y, Cheung F, Nagamatsu T (2011). Hepatoprotective effect and its possible mechanism of Coptidis rhizoma aqueous extract on carbon tetrachloride-induced chronic liver hepatotoxicity in rats. J Ethnopharmacol 138(3): 683-690. DOI: 10.1016/j.jep.2011.09.032.

Fernandez-Panchon MS, Villano D, Troncoso AM, Garcia-Parrilla MC (2008). Antioxidant activity of phenolic compounds: from in vitro results to in vivo evidence. Crit Rev Food Sci Nutr 48(7): 649-671. DOI: $10.1080 / 10408390701761845$.

French SW, Miyamoto K, Ohta Y, Geoffrion Y (1988). Pathogenesis of experimental alcoholic liver disease in the rat. Methods. Achiev Exp Pathol 13: 181-207.

Gelissen IC, Brodie B, Eastwood MA (1994). Effect of Plantago ovata (psyllium) husk and seeds on sterol metabolism: studies in normal and ileostomy subjects. Am J Clin Nutr 59: 395-400. DOI: 10.1093/ajen/59.2.395.

Gowda S, Desai PB, Hull VV, Math AA, Vernekar SN, Kulkarni SS (2009). A review on laboratory liver function tests. Pan Afr Med J 3: 17 .
Huang HL, Wang YJ, Zhang QY, Liu B, Wang FY, Li JJ, Zhu RZ (2012). Hepatoprotective effects of baicalein against $\mathrm{CCl}_{4}{ }_{4}$ induced acute liver injury in mice. World J Gastroenterol 18(45): 6605-6613. DOI: 10.3748/wjg.v18.i45.6605.

Hung MY, Fu TY, Shih PH, Lee CP, Yen GC (2006). Du-Zhong (Eucommia ulmoides Oliv.) leaves inhibits CCl4-induced hepatic damage in rats. Food Chem Toxicol 44(8): 1424-1431. DOI: 10.1016/j.fct.2006.03.009.

Kardosova A, Machova E (2006). Antioxidant activity of medicinal plant polysaccharides. Fitoterapia 77(5): 367-373. DOI: 10.1016/j. fitote.2006.05.001.

Kil HY, Seong ES, Ghimire BK, Chung IM, Kwon SS, Goh EJ et al. (2009). Antioxidant and antimicrobial activities of crude sorghum extract. Food Chem 115(4): 1234-1239. DOI: 10.1016/j. foodchem.2009.01.032.

Li S, Tan HY, Wang N, Zhang ZJ, Lao L, Wong CW, Feng Y (2015). The role of oxidative stress and antioxidants in liver diseases. Int J Mol Sci 16(11): 26087-26124. DOI: 10.3390/ijms161125942.

Pandey KB, Rizvi SI (2009). Plant polyphenols as dietary antioxidants in human health and disease. Oxid Med Cell Longev 2(5): 270-278. DOI: 10.4161/oxim.2.5.9498.

Park YS, Jung ST, Kang SG, Heo BG, Arancibia-Avila P, Toledo F et al. (2008). Antioxidants and proteins in ethylene-treated kiwifruits. Food Chem 107(2): 640-648. DOI: 10.1016/j. foodchem.2007.08.070.

Patel MK, Mishra A, Jha B (2016). Non-targeted Metabolite Profiling and Scavenging Activity Unveil the Nutraceutical Potential of Psyllium (Plantago ovata Forsk). Front Plant Sci 7: 431. DOI: 10.3389/fpls.2016.00431.

Rodriguez-Cabezas ME, Galvez J, Camuesco D, Lorente MD, Concha A, Martinez-Augustin O, et al. (2003). Intestinal antiinflammatory activity of dietary fiber (Plantago ovata seeds) in HLA-B27 transgenic rats. Clin Nutr 22: 463-471. DOI: 10.1016/ s0261-5614(03)00045-1.

Singal AK, Jampana SC, Weinman SA (2011). Antioxidants as therapeutic agents for liver disease. Liver Int 31(10): 14321448. DOI: 10.1111/j.1478-3231.2011.02604.x.

Singleton VL, Orthofer R, Lamuela-Raventos RM (1999). Analysis of total phenols and other oxidation substrates and antioxidants by means of Folin-Ciocalteu reagent. Methods Enzymol, 299(27): 152-178. DOI: 10.1016/S0076-6879(99)99017-1.

Teselkin YO, Babenkova IV, Kolhir VK, Baginskaya AI Tjukavkina NA, Kolesnik YA, et al. (2000). Dihydroquercetin as a means of antioxidative defence in rats with tetrachloromethane hepatitis. Phytother Res 14(3): 160-162. DOI: 10.1002/ (sici)1099-1573(200005)14:3<160::aid-ptr555>3.0.co;2-y.

Umamaheswari M, Chatterjee TK (2008). In vitro antioxidant activities of the fractions of Coccinia grandis L. leaf extract. Afr J Tradit Complement Altern Med 5: 61-73. DOI: 10.4314/ajtcam. v5i1.31258.

Wahid A, Hamed AN, Eltahir HM, Abouzied MM (2016). Hepatoprotective activity of ethanolic extract of Salix subserrata against $\mathrm{CCl}_{4}$-induced chronic hepatotoxicity in rats. BMC Complement Altern Med 16: 263. DOI: 10.1186/s12906-0161238-2. 